\title{
Orbital selective overlayer-substrate hybridization in a Pb monolayer on $\mathrm{Ag}(111)$
}

\author{
Christian R. Ast, $, 2, *$ Daniela Pacilé,,${ }^{1,3}$ Marco Papagno, ${ }^{1,3}$ Thomas Gloor, ${ }^{4}$ Frédéric Mila, ${ }^{4}$ Stephan Fedrigo,,${ }^{1}$ Gero Wittich, ${ }^{2}$ \\ Klaus Kern, ${ }^{1,2}$ Harald Brune, ${ }^{1}$ and Marco Grioni ${ }^{1}$ \\ ${ }^{1}$ Ecole Polytechnique Fédérale de Lausanne (EPFL), Institut de Physique des Nanostructures, CH-1015 Lausanne, Switzerland \\ ${ }^{2}$ Max-Planck-Institut für Festkörperforschung, 70569 Stuttgart, Germany \\ ${ }^{3}$ INFN and Universitá degli studi della Calabria, 87036 Rende, Cosenza, Italy \\ ${ }^{4}$ Ecole Polytechnique Fédérale de Lausanne (EPFL), Institut de Théorie des Phénomènes Physiques, CH-1015 Lausanne, Switzerland
}

(Received 16 January 2006; revised manuscript received 5 May 2006; published 23 June 2006)

\begin{abstract}
The electronic structure of a $\mathrm{Pb}$ overlayer on $\mathrm{Ag}(111)$ has been studied by angle-resolved photoemission spectroscopy. We identify three $p$ bands. While the $p_{x y}$ bands are sharp and closely resemble the corresponding bands of a free-standing $\mathrm{Pb}$ layer, the $p_{z}$ band is strongly spread in energy and momentum. Tight-binding calculations of the electronic structure combined with structural calculations using the embedded atom method indicate a symmetry dependent hybridization with the $\mathrm{Ag}(111)$ surface. We propose a symmetry selective, surface state mediated coupling to the bulk continuum which results in a broadened line shape for the $p_{z}$ band only.
\end{abstract}

DOI: 10.1103/PhysRevB.73.245428

\section{INTRODUCTION}

The electronic structure of low-dimensional metal-onmetal systems has attracted a lot of attention due to the appearance of peculiar instabilities such as charge density waves and Peierls transitions. ${ }^{1,2}$ Further, in monolayer films with long period superstructures the back folding of the band structure has been observed. ${ }^{3-6}$ Among the metal-on-metal systems with long period superstructures one distinguishes networks of surface partial dislocations with localized stacking transitions ${ }^{7}$ from moiré patterns where these transitions occur homogeneously over the entire unit cell. ${ }^{8,9}$ For the electronic structure, particularly moiré patterns have been of interest, since in order for them to be formed, the lateral stiffness of the film has to be large compared to the atomic corrugation of the substrate binding potential. Therefore, to first approximation, the vertical interaction is weak, and the films display almost the electronic structure of a freestanding monolayer..$^{10}$ However, as we will show in the following, the remaining interaction with the substrate can be strongly symmetry dependent and mediated by a surface state. Another consequence of the weak vertical binding of moire structures is that the adsorbates forming such films often act as surfactants in homoepitaxy, ${ }^{11-14}$ which requires the evaporated atoms to be able to penetrate and diffuse under the surfactant layer. ${ }^{15}$

Here we report an angle-resolved photoelectron spectroscopy (ARPES) study of the electronic states of a $\mathrm{Pb}$ monolayer on $\operatorname{Ag}(111)$, a model system with a long range ordered moiré-type superstructure. We show that due to a symmetry selective hybridization with the substrate the $\mathrm{Pb}$ states with $p_{z}$ character interact with the Ag bulk through a coupling to the $\operatorname{Ag}(111)$ surface state, and that the coupling is much stronger than for $\mathrm{Pb}$ states with $p_{x y}$ character. This interaction leads to a selective breakdown of the overlayer band structure. The interpretation is based on structure calculations using the embedded atom method (EAM) (Ref. 16) combined with tight-binding calculations for the electronic structure.
PACS number(s): 73.20.At, 79.60.-i

\section{EXPERIMENT}

The samples were prepared in ultra high vacuum by successive sputter/annealing cycles of the $\operatorname{Ag}(111)$ crystal to clean the surface followed by the deposition of a monolayer of $\mathrm{Pb}$ and post annealing to achieve structural equilibrium. The annealing temperature for the clean $\mathrm{Ag}(111)$ crystal was $800 \mathrm{~K}$, while the post-annealing temperature with the $\mathrm{Pb}$ monolayer was $500 \mathrm{~K}$. The structural quality has been verified by low energy electron diffraction (LEED) which showed a typical moiré diffraction pattern. ${ }^{17}$ The base pressure was $<1 \times 10^{-10} \mathrm{mbar}$. ARPES measurements were done at 77 and at $300 \mathrm{~K}$ using HeI radiation $(21.2 \mathrm{eV})$. The energy resolution of the spectrometer was $10 \mathrm{meV}$ and the angular resolution was better than $1^{\circ}$.

\section{RESULTS}

A topographic image measured by scanning tunneling microscopy of this structure in Fig. 1(a) shows atomically resolved $\mathrm{Pb}$ atoms in a hexagonal lattice. The lattice mismatch between the $\mathrm{Pb}$ monolayer and the $\mathrm{Ag}(111)$ substrate, accompanied by the rather strong lateral bonding within the overlayer, induces a long range ordered hexagonal moiré pattern. A Fourier transform of this image (not shown here) reveals that the in-plane lattice constants of $\mathrm{Pb}$ and $\mathrm{Ag}(111)$ at the surface are $3.51 \AA$ (Pb bulk: $3.50 \AA$ ) and $2.89 \AA$, respectively. We find two equivalent $\mathrm{Pb}$ domains rotated by $\pm 4.5^{\circ}$ with respect to the Ag surface lattice, of which one is displayed in Fig. 1(a). Figure 1(b) shows a schematic of the undistorted $\mathrm{Pb}$ and $\mathrm{Ag}$ lattices. The small hexagon in the middle is the hexagonal unit cell of the nonmodulated $\mathrm{Pb}$ lattice while the larger hexagon represents the hexagonal unit cell of the moiré reconstruction which is denoted by $(\sqrt{28}$ $\times \sqrt{28}) R 19.1^{\circ}$ with respect to the $\mathrm{Ag}$ substrate and $(\sqrt{19}$ $\times \sqrt{19}) R 23.4^{\circ}$ with respect to the $\mathrm{Pb}$ lattice. The corresponding surface Brillouin zones are displayed in Fig. 1(c). Dashed lines show the $\mathrm{Ag}(111)$ surface Brillouin zone while solid lines represent the Brillouin zones of the two rotated $\mathrm{Pb}$ do- 

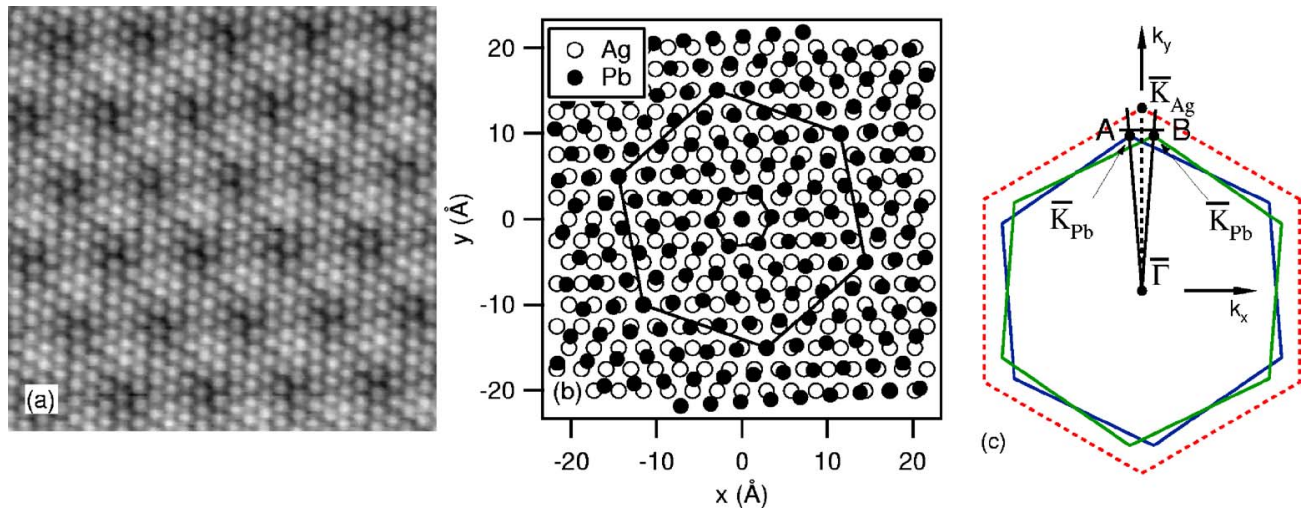

FIG. 1. (Color online) (a) STM topography of the moiré structure. The Pb atoms are atomically resolved. (b) The undistorted structure. (c) Schematic of the surface Brillouin zones (SBZ) for $\mathrm{Ag}(111)$ (dashed lines) as well as for the two rotated Pb overlayer domains (solid lines). The symmetry points have the subscripts $\mathrm{Ag}$ and $\mathrm{Pb}$, respectively.

mains. The schematic indicates that for each Brillouin zone the high symmetry lines $\bar{\Gamma} \overline{\mathrm{K}}$ point in slightly different directions. We therefore denote the high symmetry lines for the silver and the lead lattice as $\bar{\Gamma} \overline{\mathrm{K}}_{\mathrm{Ag}}$ and $\bar{\Gamma} \overline{\mathrm{K}}_{\mathrm{Pb}}$, respectively. This should be visible in the ARPES data.

The modulation of the atomic positions in the first layers at the surface has been modeled by molecular dynamic simulations within the embedded atom method (EAM). The inplane positions of the $\mathrm{Pb}$ atoms obtained from the ideal undistorted structure in Fig. 1(b) were used as input parameters. The $\mathrm{Ag}-\mathrm{Ag}$ and the $\mathrm{Pb}-\mathrm{Pb}$ interactions have been taken from Refs. 16 and 18, respectively, while the $\mathrm{Pb}-\mathrm{Ag}$ has been approximated by a combination of the two monoatomic potentials in the way proposed by Johnson. ${ }^{19}$ In order to reproduce the $(4 \times 4)$ superstructure of $\mathrm{Pb}$ on $\mathrm{Cu}(111)$ (Ref. 20) the $\mathrm{Pb}-\mathrm{Cu}$ potential adapted from Ref. 19 had to be increased by a factor of three, which has been adopted for the $\mathrm{Pb} / \mathrm{Ag}(111)$ structure. A 22-layer slab of silver covered by a monolayer of $\mathrm{Pb}$ has been used with the two bottom layers frozen. The result for the first two layers can be seen in Fig. 2 as a side view. The atomic positions are modulated mainly perpendicular to the surface. While the out-of-plane modulation of atom positions in the $\mathrm{Pb}$ layer is with $0.09 \AA$ relatively

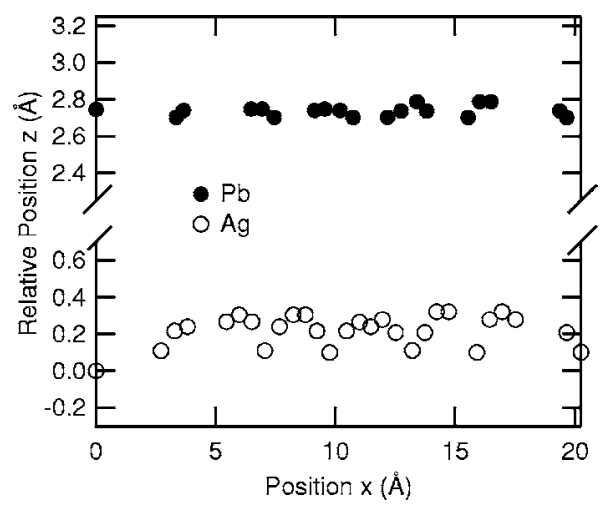

FIG. 2. Side view of the calculated atom positions near the surface. The $y$ axis is compressed to enhance the differences in atom positions. The scaling is the same for the upper and the lower part. small, the modulation of the $\mathrm{Ag}$ atoms in the top layer amounts to $0.32 \AA$. A qualitatively similar result has been obtained for $\mathrm{a} \mathrm{Pb}$ overlayer on $\mathrm{Cu}(111){ }^{21}$ For the $\mathrm{Pb} / \mathrm{Cu}(111)$ system, the $\mathrm{Pb}$ overlayer induced modulation of the substrate surface has been proposed to be related to the surface-active role of $\mathrm{Pb}$. Due to the peculiar atom positions this is referred to as inverse corrugation, which results in a reduced buckling of the $\mathrm{Pb}$ overlayer. ${ }^{20}$ Because of the similarities in the two systems, we propose a very similar mechanism for the $\mathrm{Pb}$ monolayer on $\mathrm{Ag}(111)$.

The experimental band structure of $\mathrm{Pb} / \mathrm{Ag}(111)$ along the $\bar{\Gamma} \overline{\mathrm{K}}_{\mathrm{Pb}}$ line is illustrated as a stack of energy distribution curves (EDC) in Fig. 3. The EDCs have been measured in one degree steps starting with the $\bar{\Gamma}$ point (normal emission) at the bottom of the plot. The peaks associated with the $\mathrm{Pb}$ monolayer band structure are indicated by vertical tick marks indicating the dispersion. The same data is displayed as an intensity image in Fig. 4(a). The intensity is on a linear scale with black corresponding to highest photoemission intensity. The strong emission feature which disperses from $-3.7 \mathrm{eV}$ at about $0.8 \AA^{-1}$ to the Fermi level, as indicated by the arrow, is the $s p$-derived Ag bulk band. The other emission features are related to the electronic structure of the $\mathrm{Pb}$ monolayer. We identify three bands within $2 \mathrm{eV}$ binding energy of which two bands have minima at $-0.8 \mathrm{eV}$ as well as $-2 \mathrm{eV}$ near $\overline{\mathrm{K}}_{\mathrm{Pb}}$ and one broad band is located near $\bar{\Gamma}$. Comparing the experimental band structure with the tight-binding calculation of a free-standing $\mathrm{Pb}$ monolayer [see Fig. 4(c)], we can identify the two bands near $\overline{\mathrm{K}}_{\mathrm{Pb}}$ as $p_{x y}$ bands and the broad band as $p_{z}$ band.

Figure 4(b) illustrates the dispersion of these bands in a direction perpendicular to $\bar{\Gamma} \overline{\mathrm{K}}_{\mathrm{Ag}}$ [line $\mathrm{AB}$ in Fig. 1(c) and tilted line in Fig. 4(a) near $\overline{\mathrm{K}}_{\mathrm{Pb}}$ ]. It shows two pairs of bands due to the two possible orientations of $\mathrm{Pb}$ domains, with maxima along the $\bar{\Gamma} \overline{\mathrm{K}}_{\mathrm{Pb}}$ lines. These bands cross at the $\bar{\Gamma} \overline{\mathrm{K}}_{\mathrm{Ag}}$-line. The coexistence of the two domains explains the apparent splitting of the $p_{x y}$ band which is observed near the $\overline{\mathrm{K}}_{\mathrm{Pb}}$ point in Fig. 4(a). 


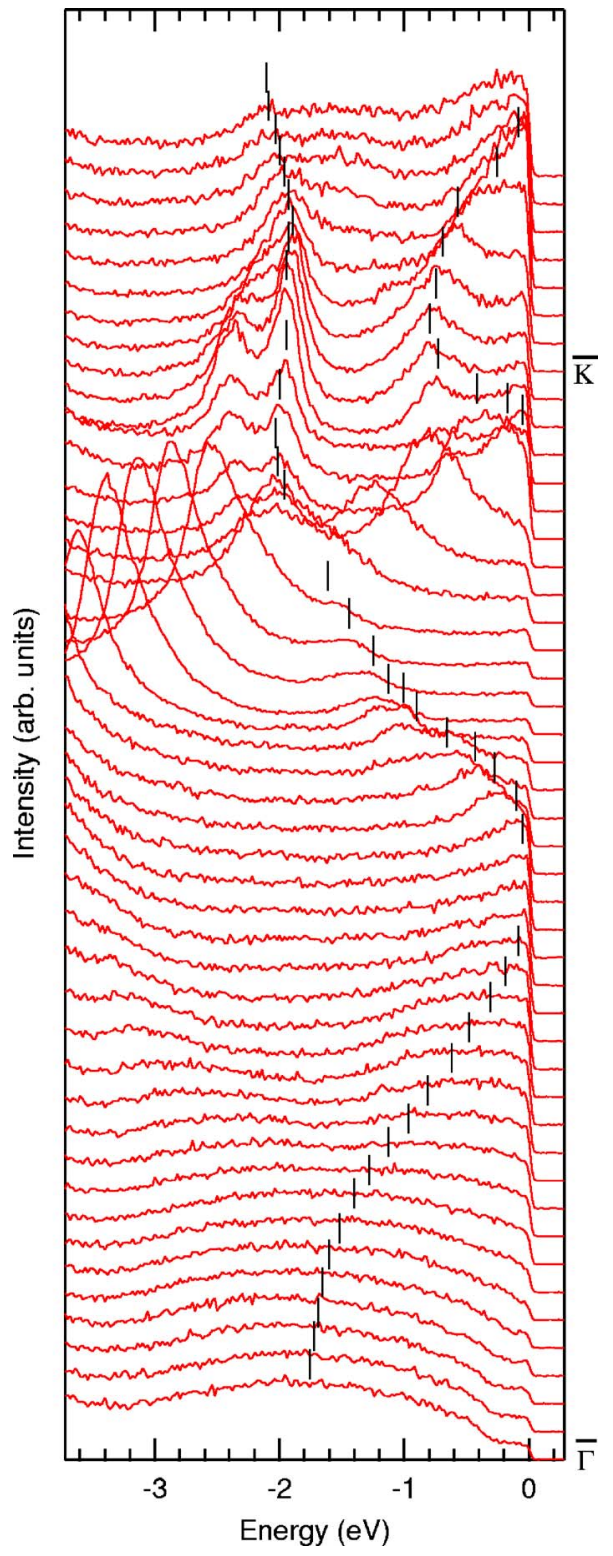

FIG. 3. (Color online) Stack of energy distribution curves of the $\mathrm{Pb}$ monolayer on $\operatorname{Ag}(111)$ along $\bar{\Gamma} \overline{\mathrm{K}}$. The vertical tick marks indicate the dispersion of the bands associated with the $\mathrm{Pb}$ monolayer.

\section{DISCUSSION}

The striking aspect of the ARPES results in Figs. 3 and 4 is the very broad emission feature of the $p_{z}$ band dispersing from $\bar{\Gamma}$ toward the Fermi level near the $p_{x y}$ band. Its spectral weight is so much spread in energy and momentum that the dispersion is barely recognizable. To better visualize the broadening of the $p_{z}$ band and the $p_{x y}$ bands, Fig. 5(a) shows three energy distribution curves (EDC) as dotted lines at selected momentum values along the $\bar{\Gamma} \overline{\mathrm{K}}_{\mathrm{Pb}}$ line. The inelastic background has been subtracted from these spectra. The fit is shown as a solid line with the Lorentzian components as dashed lines. In the upper graph $\left(k_{\|}=0.88 \AA^{-1}\right)$, the $p_{x y}$ band appears as a double peak structure around $-1.1 \mathrm{eV}$ due to the two coexisting domains. The two peaks have Lorentzian widths of 0.19 and $0.29 \mathrm{eV}$. The middle graph displays an EDC measured at $k_{\|}=0.44 \AA^{-1}$, just before the Fermi level crossing of the $p_{z}$ band, which appears as a broad peak. The Lorentzian width of this peak is $1.86 \mathrm{eV}$, much broader than the widths of the features associated with the $p_{x y}$ band. A momentum distribution curve (MDC) along the $\bar{\Gamma} \overline{\mathrm{K}}_{\mathrm{Ag}}$ line at an energy of $-0.48 \mathrm{eV}$ [Fig. 5(b)] shows two structures corresponding to the $p_{z}$ and $p_{x y}$ bands. They were fitted with Gaussian line shapes with line widths of $0.53 \pm 0.04$ and $0.11 \pm 0.03 \AA^{-1}$, respectively.

The discrepancies between the free-standing $\mathrm{Pb}$ monolayer calculation and the ARPES results point to the crucial role of the overlayer-substrate interaction in determining the nature of the electronic states at the interface. We have modeled this effect by a tight-binding calculation for the three $6 p$ orbitals of a $\mathrm{Pb}$ layer coupled to the $5 s$ orbitals of an $\mathrm{Ag}$ layer. A tight-binding approach is not expected to yield a quantitative agreement with the experiment, e.g., it fails to reproduce the gap at the $\overline{\mathrm{K}}$ point [see Figs. 4(a) and 4(c)]. Nevertheless, we find that it provides a very good qualitative indication about the origin of the phenomenon. The result of this calculation is displayed in Fig. 6. The atomic positions for each of the $19 \mathrm{~Pb}$ atoms and $28 \mathrm{Ag}$ atoms in the supercell have been taken from the EAM calculation (see Fig. 2). The diagonal energies $\mu^{\left(p_{i}\right)}$ are taken equal for all $p$ orbitals which yield two parameters $\mu^{(p)}$ and $\mu^{(s)}$. The coupling is parametrized by the tight-binding parameter $V_{s p \sigma}$. The other relevant parameters are obtained from Ref. 22, i.e., their dependence on angles and distances for the distorted structure. Figure 6(a) shows the bands for the two layers with no coupling between them. When the coupling is turned on in Fig. 6(b), the $s$ and $p_{z}$ bands change their dispersion: They hybridize and repel each other. The hybridization affects all the occupied $p_{z}$ states, since the two bands run parallel over a large portion of the Brillouin zone (BZ). As a result, the $s$ band moves above the Fermi level and the $p$ bands to higher binding energies. The $p_{z}$ band now carries about 20\% of $s$ character. Although slightly smaller than the typical value, ${ }^{22}$ the parameter $V_{s p \sigma}=0.46 \mathrm{eV}$ was chosen so that the dispersion of the hybridized $p_{z}$ band lies within the broad experimental feature. The hybridization of the $s$ band with the occupied $p_{x y}$ bands is considerably smaller both because of the larger energy separation, and of symmetry considerations. Here the $p_{x y}$ bands fully retain their $p$ character.

The line thickness in Figs. 6(a) and 6(b) is proportional to the spectral weight, which is partially transferred from the bands of the unmodulated structure to those corresponding to the modulated atom positions, where the thinnest lines actually carry a vanishingly small spectral weight. ${ }^{23}$ Clearly, most of the spectral weight of the unperturbed bands remains with the original dispersion. We conclude that the perturbing potential of the modulation which is perceived by the electrons in the $\mathrm{Pb}$ overlayer is small compared to the potential of the unmodulated, flat monolayer. This is further supported by the experimental band dispersion, which follows the BZ of the unmodulated $\mathrm{Pb}$ unit cell. Therefore, the moiré superstructure per se is not a major factor in the breakdown of the $p_{z}$-band dispersion. A similar conclusion is arrived at for $\mathrm{Ag} / \mathrm{Cu}(111)$ where the moiré modulation does not change the Brillouin zone. ${ }^{24}$ 

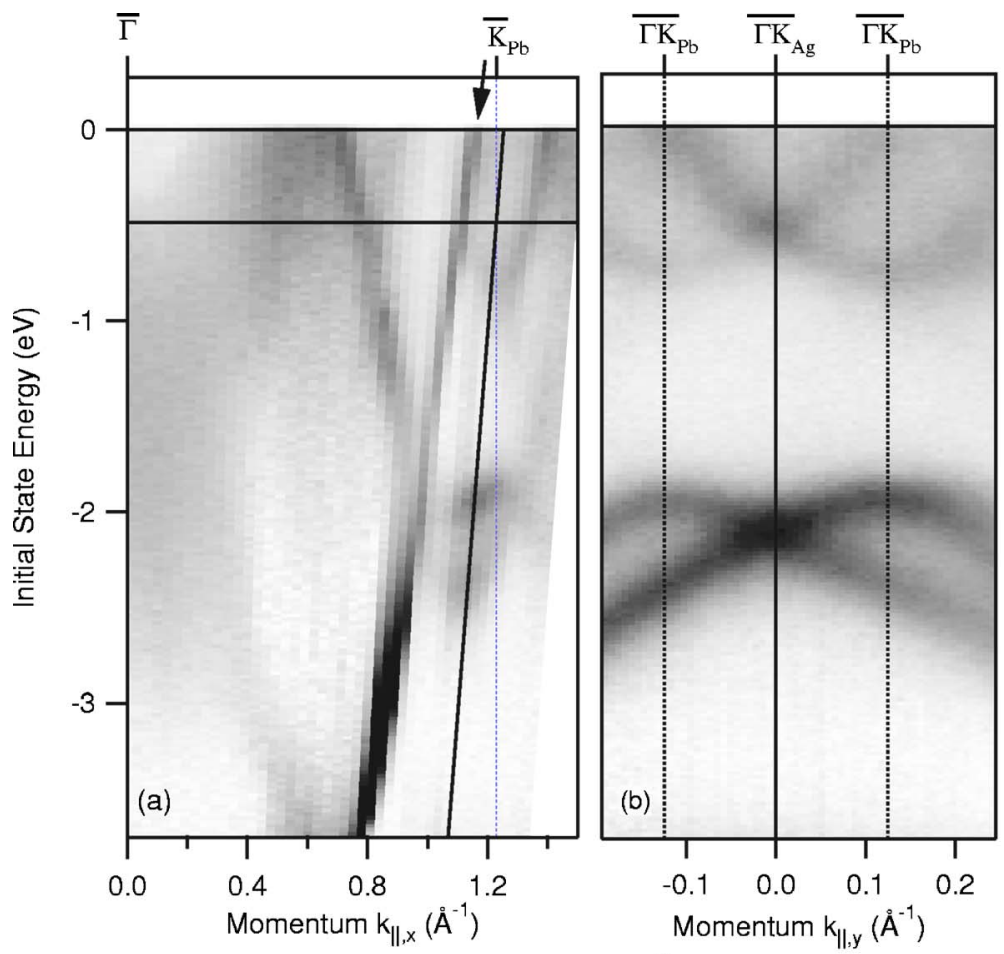

FIG. 4. (Color online) (a) Band structure measured along $\bar{\Gamma} \overline{\mathrm{K}}$. (b) Slice perpendicular to $\bar{\Gamma} \overline{\mathrm{K}}$ along tilted line in (a). (c) Simple 2D tightbinding calculations for $p$ bands in a hexagonal lattice. (d) Outline of the projected band structure of $\operatorname{Ag}(111)$.
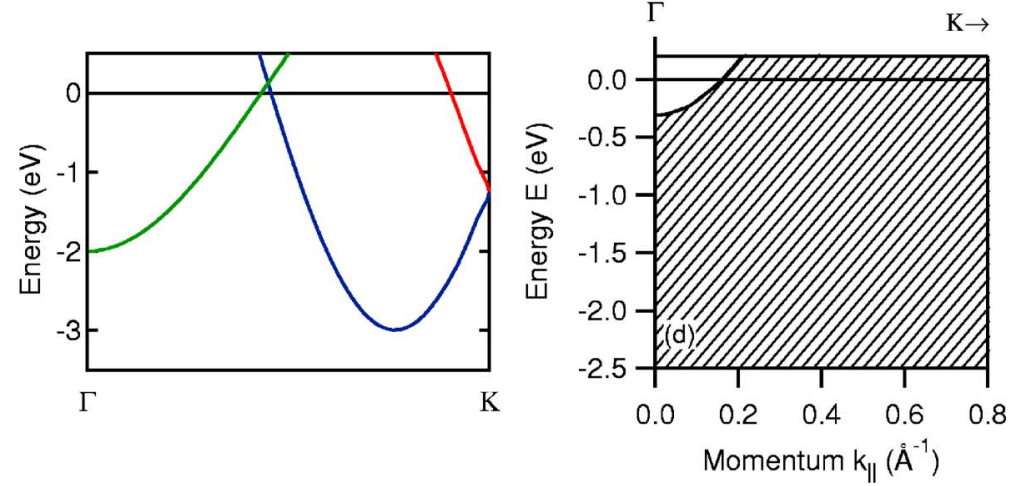

The large unit cell of the reconstruction introduces another possible source of spectral broadening. The $\mathrm{Pb}$ adatoms occupy several inequivalent adsorption sites. This modulates the diagonal energies which we have supposed constant in the tight-binding (TB) calculation. Modulating the diagonal energies for the $p$ orbitals introduces disorder and actually broadens the bands (not shown). Nevertheless, we find that the effect becomes comparable to the ARPES measurements only for energy variations similar to the experimental linewidth $(\sim 2 \mathrm{eV})$, which is well beyond what is to be expected from the EAM calculations.

From the presented results and the preceding discussion we propose a different scenario. Because of the closer proximity in energy, and possibly the more favorable orientation of the $p_{z}$ orbitals, the $p_{z}$ band of the overlayer is more likely to interact with the $s p$ derived surface state of the $\operatorname{Ag}(111)$ substrate than the $p_{x y}$ bands. Through the hybridization with the surface state the $p_{z}$ band acquires some $s$ character and a longer penetration depth into the bulk. Comparing Figs. 4(a) and $4(\mathrm{~d})$ we find that the hybrid band resides within the projected $\mathrm{Ag}(111)$ bulk band structure so that its interaction with the bulk electronic states spreads spectral weight around the band dispersion. For a given $k_{\|}$value, this is analogous to the problem of an impurity interacting with a continuum. Apart from possible band structure effects the resulting energy broadening is then proportional to the effective hybridization $V^{2}$ between the hybrid surface state and the bulk continuum. ${ }^{25}$ In reality, the spectral distribution has an upper bound given by the gap in the projected band stucture [Fig. 2(d)]. This can be seen more clearly in the EDC measured at $\bar{\Gamma}$ in the lower panel of Fig. 5(a). The photoemission intensity of the $p_{z}$ band is clearly truncated as it enters the projected band gap. As a consequence, the image showing the spectral weight distribution [see Fig. 4(a)] clearly outlines the projected energy gap [see Fig. 4(d)]. The $p_{x y}$ bands also reside within the projected $\operatorname{Ag}(111)$ bulk band structure but these states interact only weakly with the surface state and with the bulk states. These states are therefore more localized within the $\mathrm{Pb}$ overlayer leading to a well-defined peak in the photoemission spectra.

\section{CONCLUSION}

In conclusion, we find for the monolayer of $\mathrm{Pb}$ on $\operatorname{Ag}(111)$ that electrons in states with different symmetries 

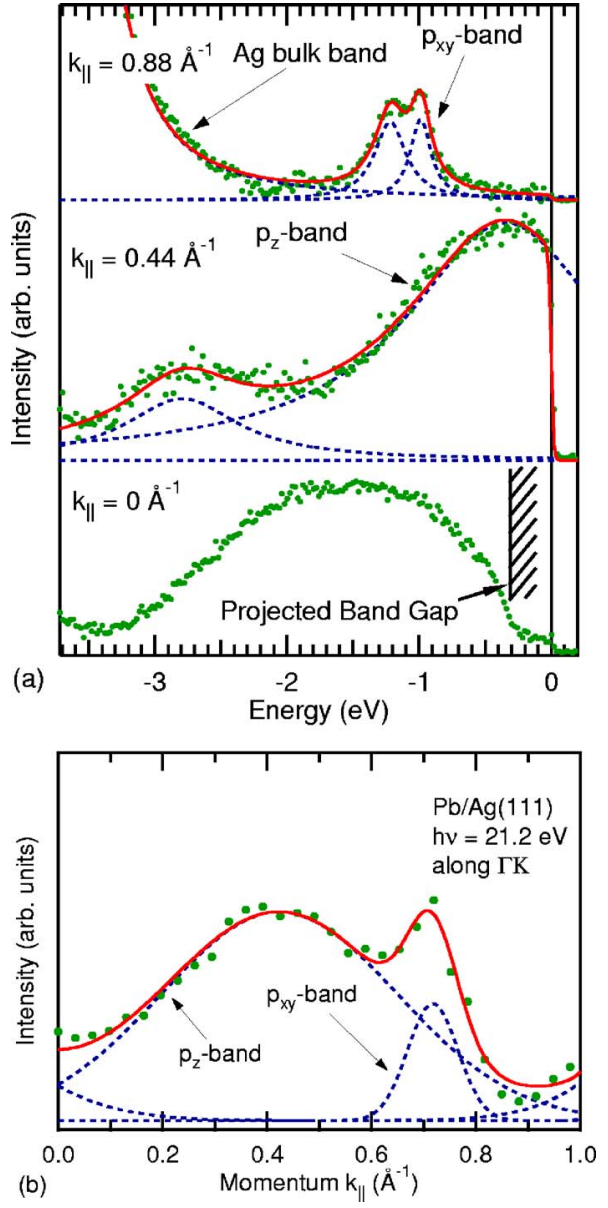

FIG. 5. (Color online) (a) Energy distribution curves measured at three different wave vectors along the $\bar{\Gamma} \overline{\mathrm{K}}_{\mathrm{Pb}}$ line. (b) Momentum distribution curve at $-0.48 \mathrm{eV}$ showing the $p_{z}$ band and a $p_{x y}$ band [see horizontal line in Fig. 4(a)]. The dots show the data, the solid lines show the fit, while the dashed lines indicate the components of the fit function.

behave differently within the same potential, leading to a selective breakdown of the interface band structure. The $p_{z}$ states hybridize with the substrate much more effectively than the $p_{x y}$ states. Interestingly, this leads to a broadening of the peak more than a substantial change in dispersion. This is reflected in the much broader line width of the $p_{z}$ band which is directly proportional to their relative hybridization with the bulk bands. The photoemission spectra show that the broad $p_{z}$ band outlines, as expected, the projected bulk band structure near $\bar{\Gamma}$. Combining this with the tight-binding calculation which shows a selective coupling of the $p$ bands to the $\operatorname{Ag}(111)$ surface state we conclude that the $\mathrm{Ag}$ surface state mediates the coupling to the bulk continuum. A more elaborate model like a first-principles slab calculation would certainly provide a more detailed description of the overlayer
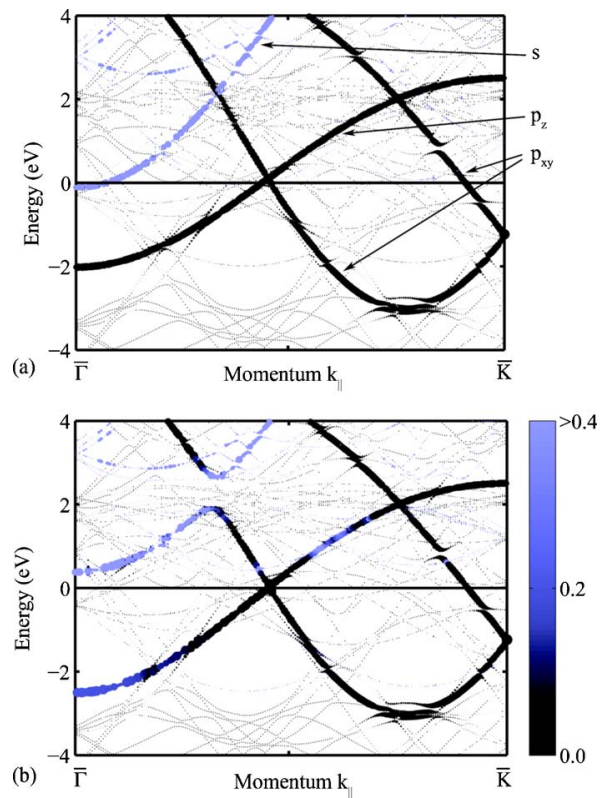

FIG. 6. (Color online) Tight binding calculation of a Pb layer with $p$ bands and an Ag layer with an $s$ band, (a) without and (b) with coupling between layers $\left(V_{s p \sigma}=0.46 \mathrm{eV}\right)$. The atom positions have been taken from the structure calculation. The line thickness is proportional to the spectral weight. The color coding indicates the character with (light gray) and black for pure $s$ and $p$ characters, respectively. The color (grayscale) bar in (b) indicates the amount of $s$ character after hybridization.

band dispersion. Such a calculation would be computationally quite costly given the large unit cell.

Similar results for other systems suggest that such extremely orbital-selective hybridization of interface states with the substrate is a more general phenomenon. The broadening of the $p_{z}$ states went unnoticed for a $\mathrm{Pb}$ monolayer on $\mathrm{Cu}(111)$ in Ref. 10, but from an analysis of those data we find that this phenomenon also exists in this system, although less pronounced. The band structure is very comparable to the $\mathrm{Pb}$ monolayer on $\mathrm{Ag}(111)$. Also for a Bi monolayer on $\operatorname{Ag}(111)$ and $\mathrm{Au}(111)$ substrates we have observed a selective hybridization even though the two-dimensional band structures are qualitatively different. Only with modern ARPES and with intensity maps as displayed in Fig. 4, these kinds of effects become readily visible and many interfaces can now be reinvestigated in this perspective.

\section{ACKNOWLEDGMENTS}

We gratefully acknowledge P. Wahl, L. Vitali, and M. A. Schneider for useful discussions and help with the STM experiments. C.R.A. acknowledges funding from the EmmyNoether-Program of the Deutsche Forschungsgemeinschaft. The work at the EPFL was supported by the Swiss National Science Foundation through the MaNEP NCCR. 
*Corresponding author. Electronic address: c.ast@fkf.mpg.de

${ }^{1}$ K. Swamy, A. Menzel, R. Beer, and E. Bertel, Phys. Rev. Lett. 86, 1299 (2001).

${ }^{2}$ T. Nakagawa, H. Okuyama, M. Nishijima, T. Aruga, H. W. Yeom, E. Rotenberg, B. Kienzer, and S. D. Kevan, Phys. Rev. B 67, 241401(R) (2003).

${ }^{3}$ A. Bendounan, F. Forster, J. Ziroff, F. Schmitt, and F. Reinert, Phys. Rev. B 72, 075407 (2005).

${ }^{4}$ F. Schiller, M. Ruiz-Osés, J. Cordon, and J. E. Ortega, Phys. Rev. Lett. 95, 066805 (2005).

${ }^{5}$ A. Bendounan, F. Forster, F. Reinert, B. Kierren, Y. FagotRevurat, and D. Malterre, Phys. Rev. Lett. 96, 029701 (2006).

${ }^{6}$ F. Schiller, J. Cordon, D. Vyalikh, A. Rubio, and J. E. Ortega, Phys. Rev. Lett. 96, 029702 (2006).

${ }^{7}$ H. Brune, H. Röder, C. Boragno, and K. Kern, Phys. Rev. B 49, 2997 (1994).

${ }^{8}$ T. Wiederholt, H. Brune, J. Wintterlin, R. J. Behm, and G. Ertl, Surf. Sci. 324, 91 (1995)

${ }^{9}$ C. Günther, J. Vrijmoeth, R. Q. Hwang, and R. J. Behm, Phys. Rev. Lett. 74, 754 (1995).

${ }^{10}$ F. Baumberger, A. Tamai, M. Muntwiler, T. Greber, and J. Osterwalder, Surf. Sci. 532, 82 (2003).

${ }^{11}$ H. A. van der Vegt, J. Vrijmoeth, R. J. Behm, and E. Vlieg, Phys. Rev. B 57, 4127 (1998).

${ }^{12}$ J. Vrijmoeth, H. A. van der Vegt, J. A. Meyer, E. Vlieg, and R. J. Behm, Phys. Rev. Lett. 72, 3843 (1994).
${ }^{13}$ G. Rosenfeld, R. Servaty, Ch. Teichert, B. Poelsema, and G. Comsa, Phys. Rev. Lett. 71, 895 (1993).

${ }^{14}$ S. Oppo, V. Fiorentini, and M. Scheffler, Phys. Rev. Lett. 71, 2437 (1993).

${ }^{15}$ J. Camarero, J. Ferrón, V. Cros, L. Gómez, A. L. Vázquez de Parga, J. M. Gallego, J. E. Prieto, J. J. de Miguel, and R. Miranda, Phys. Rev. Lett. 81, 850 (1998).

${ }^{16}$ S. M. Foiles, M. I. Baskes, and M. S. Daw, Phys. Rev. B 33, 7983 (1986).

${ }^{17}$ K. J. Rawlings, M. J. Gibson, and P. J. Dobson, J. Phys. D 11, 2059 (1978).

${ }^{18}$ D. Y. Sun, Y. Xiang, and X. G. Gong, Philos. Mag. A 79, 1953 (1999).

${ }^{19}$ R. A. Johnson, Phys. Rev. B 39, 12554 (1989).

${ }^{20} \mathrm{~S}$ Müller, J. E. Prieto, C. Rath, L. Hammer, R. Miranda, and K. Heinz, J. Phys.: Condens. Matter 13, 1793 (2001).

${ }^{21}$ C. Nagl, M. Schmid, and P. Varga, Surf. Sci. 369, 159 (1996).

${ }^{22}$ W. A. Harrison, Electronic Structure and the Properties of Solids (Dover, New York, 1989).

${ }^{23}$ J. Voit, L. Perfetti, F. Zwick, H. Berger, G. Margaritondo, G. Grüner, H. Höchst, and M. Grioni, Science 290, 501 (2000).

${ }^{24}$ A. Bendounan, F. Forster, J. Ziroff, F. Schmitt, and F. Reinert, Phys. Rev. B 72, 075407 (2005).

${ }^{25}$ H.-V. Roy, J. Boschung, F. Patthey, P. Fayet, W.-D. Schneider, and B. Delley, Phys. Rev. Lett. 70, 2653 (1993). 